\title{
Unifying Hyper and Epistemic Temporal Logics
}

\author{
Laura Bozzelli ${ }^{1}$, Bastien Maubert ${ }^{2}$, and Sophie Pinchinat ${ }^{3}$ \\ 1 UPM, Madrid, Spain. \\ ${ }^{2}$ LORIA - CNRS / Université de Lorraine, Nancy, France. \\ ${ }^{3}$ IRISA, Université de Rennes 1, France.
}

\begin{abstract}
In the literature, two powerful temporal logic formalisms have been proposed for expressing information-flow security requirements, that in general, go beyond regular properties. One is classic, based on the knowledge modalities of epistemic logic. The other one, the so-called hyper logic, is more recent and subsumes many proposals from the literature. In an attempt to better understand how these logics compare with each other, we consider the logic $\mathrm{KCTL}^{*}$ (the extension of $\mathrm{CTL}^{*}$ with knowledge modalities and synchronous perfect recall semantics) and HyperCTL*. We first establish that $\mathrm{KCTL}^{*}$ and HyperCTL* are expressively incomparable. Then, we introduce a natural linear past extension of HyperCTL* ${ }^{*}$, called HyperCTL $L_{l p}^{*}$, that unifies $\mathrm{KCTL}^{*}$ and HyperCTL*. We show that the model-checking problem for HyperCTL $L_{l p}^{*}$ is decidable, and we provide its exact computational complexity in terms of a new measure of path quantifiers' alternation. For this, we settle open complexity issues for unrestricted quantified propositional temporal logic.
\end{abstract}

\section{Introduction}

Temporal logics provide a fundamental framework for the description of the dynamic behavior of reactive systems, and they usually support the successful model-checking approach to automatically verify complex finite-state systems.

Classic regular temporal logics, such as standard LTL [21] or the more expressive $\mathrm{CTL}^{*}$ [10], lack mechanisms to relate distinct paths or executions of a system. These mechanisms are required to formally express information-flow security properties which specify how information may propagate from inputs to outputs, such as non-interference [12] or opacity [5]. In the literature, two powerful temporal logic formalisms have been proposed for expressing such security requirements that, in general, go beyond regular properties.

One is classical and is based on the extension of temporal logic with the knowledge modalities of epistemic logic [11], which relate paths that are observationally equivalent for a given agent. A classic instance is $\mathrm{KCTL}^{*}$, the extension of $\mathrm{CTL}^{*}$ with knowledge modalities under the synchronous perfect recall semantics (where an agent remembers the whole sequence of its observations, and observations are time-sensitive) $[14,24,22,8]$. This logic and its linear-time

We acknowledge financial suppport from ERC project EPS 313360. 
fragment, KLTL, have been used to specify secrecy policies that involve sets of execution traces sharing some similar information $[1,13,3]$.

In the second, more recent, framework [7] one can express properties of sets of execution traces, known as hyperproperties; these are useful to formalize security policies, such as non-interference [12] and observational determinism [18]. The general hyper logical framework introduced in [7] is based on a second-order logic for which model-checking is undecidable. More recently, fragments of this logic have been introduced [6], namely the logics HyperCTL* and HyperLTL, for which model checking is decidable. These logics extend $\mathrm{CTL}^{*}$ and $\mathrm{LTL}$ in a simple and natural way by allowing explicit and simultaneous quantification over multiple paths. In [6], an extension of the semantics of HyperCTL* and HyperLTL is also considered. In this setting, a formula can refer to propositions which extend the alphabet AP of the model $K$. Then, the path quantification ranges over all the traces on the augmented alphabet whose projections over AP correspond to the execution traces of $K$. Within this affected generalization, KLTL can be effectively expressed in HyperLTL [6]. The logic HyperCTL* also generalizes the temporal logic secLTL, introduced in [9]. Other logics for hyperproperties were introduced in [19] but no general approach to verifying such logics exists.

Contribution. Our first contribution in this paper is the comparison of the expressive power of hyper temporal logics and epistemic temporal logics. We establish by formal non-trivial arguments that HyperCTL* and $\mathrm{KCTL}^{*}$ are expressively incomparable.

As a second contribution, we unify HyperCTL* and $\mathrm{KCTL}^{*}$ by extending HyperCTL* with new logical features which provide very natural modeling facilities. The proposed extension is based on two important observations: first, HyperCTL* has no explicit mechanism to refer to the past which would be useful to relate histories of different executions (paths). This ability is partially supported in $\mathrm{KCTL}^{*}$ by means of observational equivalences between path prefixes; however, such equivalences are not expressed in the logic itself but are given as separate input parameters in the model specification. On the other hand, it is well-known that temporal logics which combine both past and future temporal modalities make specifications easier to write and more natural. In particular, the linear past setting, where the history of the current situation increases with time and is never forgotten, especially suits the specification of dynamic behaviors. A relevant example is given by the logic $\mathrm{CTL}_{l p}^{*}$, a well-known equi-expressive linear past extension of $\mathrm{CTL}^{*}$ [15] obtained by adding past temporal modalities and where path quantification is 'memoryful': it ranges over paths that start at the root of the computation tree and visit the current node. The second observation is that HyperCTL* has no explicit mechanism to select, at a given non-initial instant, paths which do not visit the current node. This is clearly a strong limitation for expressing general information-flow requirements.

We remove the above two limitations of HyperCTL* by introducing both linear past modalities and the general hyper quantifier, where path quantification ranges over all the paths that start at the root of the computation tree. These new features yield a novel logic that we call HyperCTL ${ }_{l p}^{*}$. In fact, as we for- 
mally establish, the only addition of general path quantification to HyperCTL* makes the resulting logic already more expressive than HyperCTL*. However, it remains open whether both linear past and general quantification are necessary to capture all the $\mathrm{KCTL}^{*}$ definable properties. Like for the logics $\mathrm{KCTL}^{*}$ and HyperCTL*, the finite-state model-checking problem for HyperCTL $L_{l p}^{*}$ is nonelementarily decidable, and we provide the exact complexity in terms of a variant of the standard alternation depth of path quantifiers. For this, we settle complexity issues for satisfiability of full Quantified Propositional Temporal Logic (QPTL) [23]. The optimal upper bounds for full QPTL are obtained by a sophisticated generalization of the standard automata-theoretic approach for QPTL in prenex normal form [23], which exploits a subclass of parity two-way alternating word automata. Our results also improve in a meaningful way the upper bounds provided in [6] for model-checking of HyperCTL*. An extended version of this paper with all the proofs can be found in [4].

\section{Preliminaries}

Let $\mathbb{N}$ be the set of natural numbers and for all $i, j \in \mathbb{N}$, let $[i, j]:=\{h \in \mathbb{N} \mid$ $i \leq h \leq j\}$. We fix a finite set AP of atomic propositions. A trace is a finite or infinite word over $2^{\mathrm{AP}}$. For a word $w$ over some alphabet, $|w|$ is the length of $w$ $\left(|w|=\infty\right.$ if $w$ is infinite), and for each $0 \leq i<|w|, w(i)$ is the $i^{t h}$ symbol of $w$. For a $\operatorname{logic}$ formalism $\mathcal{L}$ and an $\mathcal{L}$ formula $\varphi$, the size $|\varphi|$ of $\varphi$ is the number of subformulas of $\varphi$.

Structures and tree structures. A Kripke structure (over $A P$ ) is a tuple $K=\left\langle S, s_{0}, E, V\right\rangle$, where $S$ is a set of states, $s_{0} \in S$ is the initial state, $E \subseteq S \times S$ is a transition relation such that for each $s \in S,(s, t) \in E$ for some $t \in S$, and $V: S \rightarrow 2^{\mathrm{AP}}$ is an AP-valuation assigning to each state $s$ the set of propositions in AP which hold at $s$. A path $\pi=t_{0}, t_{1}, \ldots$ of $K$ is an infinite word over $S$ such that for all $i \geq 0,\left(t_{i}, t_{i+1}\right) \in E$. For each $i \geq 0, \pi[0, i]$ denotes the prefix of $\pi$ leading to the $i^{\text {th }}$ state and $\pi[i, \infty]$ the suffix of $\pi$ from the $i^{\text {th }}$ state. A finite path of $K$ is a prefix of some path of $K$. An initial path of $K$ is a path starting from the initial state. For a (finite) path $\pi=t_{0}, t_{1}, \ldots$, the trace $V(\pi)$ of $\pi$ is $V\left(t_{0}\right), V\left(t_{1}\right), \ldots$ We say that $K=\left\langle S, s_{0}, E, V\right\rangle$ is a tree structure if $S$ is a prefixclosed subset of $\mathbb{N}^{*}, s_{0}=\varepsilon$ (the root of $K$ ), and $\left(\tau, \tau^{\prime}\right) \in E \Rightarrow \tau^{\prime}=\tau \cdot i$ for some $i \in \mathbb{N}$. States of a tree structure are also called nodes. For a Kripke structure $K$, $U n w(K)$ is the tree structure obtained by unwinding $K$ from the initial state. A tree structure is regular if it is the unwinding of some finite Kripke structure.

\subsection{Temporal logics with knowledge modalities}

We recall the non-regular extensions, denoted by $\mathrm{KCTL}^{*}$ and $\mathrm{KLTL}$, of standard $\mathrm{CTL}^{*}$ and LTL obtained by adding the knowledge modalities of epistemic logic under the synchronous perfect recall semantics $[14,24,22,8]$. Unlike the asynchronous setting, the synchronous setting can be considered time sensitive in the 
sense that it can model an observer who knows that a transition has occurred even if the observation has not changed. We fix a finite set Agts of agents.

Formulas $\varphi$ of KCTL* over Agts and AP are defined as follows:

$$
\varphi::=\top|p| \neg \varphi|\varphi \vee \varphi| \mathrm{X} \varphi|\varphi \mathrm{U} \varphi| \exists \varphi \mid \mathrm{K}_{a} \varphi
$$

where $p \in \mathrm{AP}, a \in \mathrm{Agts}, \mathrm{X}$ and $\mathrm{U}$ are the "next" and "until" temporal modalities, $\exists$ is the $C T L^{*}$ existential path quantifier, and $\mathrm{K}_{a}$ is the knowledge modality for agent $a$. We also use standard shorthands: $\forall \varphi:=\neg \exists \neg \varphi$ ("universal path quantifier"), $\mathrm{F} \varphi:=\top \mathrm{U} \varphi$ ("eventually") and its dual $\mathrm{G} \varphi:=\neg \mathrm{F} \neg \varphi$ ("always"). A formula $\varphi$ is a sentence if each temporal/knowledge modality is in the scope of a path quantifier. The logic KLTL is the LTL-like fragment of KCTL* consisting of sentences of the form $\forall \varphi$, where $\varphi$ does not contain any path quantifier.

The logic $\mathrm{KCTL}^{*}$ is interpreted over extended Kripke structures $(K, O b s)$, i.e., Kripke structures $K$ equipped with an observation map Obs : Agts $\rightarrow 2^{\mathrm{AP}}$ associating to each agent $a \in$ Agts, the set $\operatorname{Obs}(a)$ of propositions which are observable by agent $a$. For an agent $a$ and a finite trace $w \in\left(2^{\mathrm{AP}}\right)^{*}$, the $a$ observable part $O b s_{a}(w)$ of $w$ is the trace of length $|w|$ such that $O b s_{a}(w)(i)=$ $w(i) \cap \operatorname{Obs}(a)$ for all $0 \leq i<|w|$. Two finite traces $w$ and $w^{\prime}$ are (synchronously) $O b s_{a}$-equivalent if $\operatorname{Obs}_{a}(w)=O b s_{a}\left(w^{\prime}\right)$ (note that $\left.|w|=\left|w^{\prime}\right|\right)$. Intuitively, an agent $a$ does not distinguish prefixes of paths whose traces are $O b s_{a}$-equivalent.

For a $\mathrm{KCTL}^{*}$ formula $\varphi$, an extended Kripke structure $\Lambda=(K, O b s)$, an initial path $\pi$ of $K$, and a position $i$ along $\pi$, the satisfaction relation $\pi, i=_{\Lambda} \varphi$ for $\mathrm{KCTL}^{*}$ is defined as follows (we omit the clauses for the Boolean connectives):

$$
\begin{array}{ll}
\pi, i==_{\Lambda} p & \Leftrightarrow p \in V(\pi(i)) \\
\pi, i==_{\Lambda} \mathrm{X} \varphi & \Leftrightarrow \pi, i+1=_{\Lambda} \varphi \\
\pi, i==_{\Lambda} \varphi_{1} \mathrm{U} \varphi_{2} \Leftrightarrow & \Leftrightarrow \text { for some } j \geq i: \pi, j=_{\Lambda} \varphi_{2} \text { and } \pi, k \models_{\Lambda} \varphi_{1} \text { for all } i \leq k<j \\
\pi, i==_{\Lambda} \exists \varphi \Leftrightarrow & \pi^{\prime}, i=_{\Lambda} \varphi \text { for some initial path } \pi^{\prime} \text { of } K \text { s.t. } \pi^{\prime}[0, i]=\pi[0, i] \\
\pi, i==_{\Lambda} \mathrm{K}_{a} \varphi \Leftrightarrow & \Leftrightarrow \text { for all initial paths } \pi^{\prime} \text { of } K \text { such that } \\
& V(\pi[0, i]) \text { and } V\left(\pi^{\prime}[0, i]\right) \text { are } O b s_{a} \text {-equivalent, } \pi^{\prime}, i=_{\Lambda} \varphi
\end{array}
$$

We say that $(K, O b s)$ satisfies $\varphi$, denoted $(K, O b s) \models \varphi$, if there is an initial path $\pi$ of $K$ s.t. $\pi, 0=_{(K, O b s)} \varphi$. Note that if $\varphi$ is a sentence, then the satisfaction relation $\pi, 0 \models_{(K, O b s)} \varphi$ is independent of $\pi$. One can easily show that KCTL* is bisimulation invariant and, in particular, $(K, O b s) \models \varphi$ iff $(U n w(K), O b s) \models \varphi$.

Example 1. Let us consider the $\mathrm{KLTL}$ sentence $\varphi_{p}:=\forall \mathrm{XFK}_{a} \neg p$. For all observation maps Obs such that $\operatorname{Obs}(a)=\emptyset,(K, O b s) \models \varphi_{p}$ means that there is some non-root level in the unwinding of $K$ at which no node satisfies $p$. Property $\phi_{p}$ is a well-known non-regular context-free branching-time property (see e.g. [2]).

\subsection{Hyper logics}

In this section, we first recall the logics HyperCTL* and HyperLTL [6] which are non-regular extensions of CTL* and LTL with a restricted form of explicit firstorder quantification over paths. Intuitively, path variables are used to express 
linear-time properties simultaneously on multiple paths. Then, we introduce the novel logic HyperCTL $L_{l p}^{*}$, an extension of HyperCTL* obtained by adding linear past and the general hyper path quantifier. In this logic, path quantification is 'memoryful', i.e., it ranges over paths that start at the root of the computation tree (the unwinding of the Kripke structure) and either visit the current node $\tau$ (regular path quantification), or visit a node $\tau^{\prime}$ at the same level as $\tau$ (nonregular path quantification).

The logic HyperCTL* [6]. For a finite set VAR of path variables, the syntax of HyperCTL* formulas $\varphi$ over AP and VAR is defined as follows:

$$
\varphi::=\top|p[x]| \neg \varphi|\varphi \wedge \varphi| \mathrm{X} \varphi|\varphi \mathrm{U} \varphi| \exists x . \varphi
$$

where $p \in \mathrm{AP}, x \in \mathrm{VAR}$, and $\exists x$ is the hyper existential path quantifier for variable $x$. Informally, formula $\exists x . \varphi$ requires that there is an initial path $\pi$ such that $\varphi$ holds when $x$ is mapped to $\pi$, and $p[x]$ asserts that $p$ holds at the current position of the path assigned to $x$. The hyper universal quantifier $\forall x$ is defined as: $\forall x . \varphi:=\neg \exists x . \neg \varphi$. A HyperCTL* formula $\varphi$ is a sentence if each temporal modality occurs in the scope of a path quantifier and for each atomic formula $p[x], x$ is bound by a path quantifier. The logic HyperLTL is the fragment of HyperCTL* consisting of formulas in prenex form, i.e., of the form $Q_{1} x_{1} \ldots Q_{n} x_{n} . \varphi$, where $Q_{1}, \ldots, Q_{n} \in\{\exists, \forall\}$ and $\varphi$ does not contain any path quantifier.

We give a semantics for HyperCTL* that is equivalent to the one in [6] but more suitable for a linear-past generalization. HyperCTL* formulas $\varphi$ are interpreted over Kripke structures $K=\left\langle S, s_{0}, E, V\right\rangle$ equipped with a path assignment $\Pi: \mathrm{VAR} \rightarrow S^{\omega}$ associating to each variable $x \in \mathrm{VAR}$ an initial path of $K$, a variable $y \in \mathrm{VAR}$, and a position $i \geq 0$. Intuitively, $\Pi(y)$ is the current path and $i$ is the current position along the paths in $\Pi$. The satisfaction relation $\Pi, y, i \models_{K} \varphi$ is defined as follows (we omit the clauses for the Boolean connectives):

$$
\begin{aligned}
& \Pi, y, i=_{K} p[x] \quad \Leftrightarrow p \in V(\Pi(x)(i)) \\
& \Pi, y, i=_{K} \mathrm{X} \varphi \quad \Leftrightarrow \Pi, y, i+1 \models_{K} \varphi \\
& \Pi, y, i=_{K} \varphi_{1} \cup \varphi_{2} \Leftrightarrow \text { for some } j \geq i: \Pi, y, j \models_{K} \varphi_{2} \text { and } \\
& \Pi, y, k \models_{K} \varphi_{1} \text { for all } i \leq k<j \\
& \Pi, y, i=_{K} \exists x . \varphi \quad \Leftrightarrow \text { for some initial path } \pi \text { of } K \text { such that } \pi[0, i]=\Pi(y)[0, i], \\
& \Pi[x \leftarrow \pi], x, i \models \varphi
\end{aligned}
$$

where $\Pi[x \leftarrow \pi](x)=\pi$ and $\Pi[x \leftarrow \pi](y)=\Pi(y)$ for all $y \neq x$. We say that $K$ satisfies $\varphi$, written $K \models \varphi$, if there is a path assignment $\Pi$ of $K$ and $y \in \mathrm{VAR}$ such that $\Pi, y, 0 \models_{K} \varphi$. If $\varphi$ is a sentence, then the satisfaction relation $\Pi, y, 0 \models_{K} \varphi$ is independent of $y$ and $\Pi$.

Example 2. As an example of a formula expressing a non-regular requirement, we consider the HyperLTL sentence $\exists x . \exists y . p[x] \mathrm{U}((p[x] \wedge \neg p[y]) \wedge \mathrm{XG}(p[x] \leftrightarrow p[y]))$ which asserts that there are two distinct initial paths $\pi$ and $\pi^{\prime}$ and $\ell>0$ such that $p$ always holds along the prefix $\pi[0, \ell], p$ does not hold at position $\ell$ of $\pi^{\prime}$, and the valuations of $p$ along $\pi$ and $\pi^{\prime}$ coincide for all positions $j>\ell$. 
The novel logic HyperCTL $L_{l p}^{*}$. HyperCTL $L_{l p}^{*}$ formulas $\varphi$ are defined as follows:

$$
\varphi::=\top|p[x]| \neg \varphi|\varphi \wedge \varphi| \mathrm{X} \varphi\left|\mathrm{X}^{-} \varphi\right| \varphi \mathrm{U} \varphi\left|\varphi \mathrm{U}^{-} \varphi\right| \exists x . \varphi \mid \exists^{G} x . \varphi
$$

where $\mathbf{X}^{-}$and $\mathrm{U}^{-}$are the past-time counterparts of the temporal modalities $\mathrm{X}$ and $\mathrm{U}$, respectively, and $\exists^{G} x$ is the general (hyper) existential quantifier for variable $x$. We also use some shorthands: $\forall^{G} x . \varphi:=\neg \exists^{G} x . \neg \varphi$ ("general universal path quantifier"), $\mathrm{F}^{-} \varphi:=\mathrm{TU}^{-} \varphi$ ("sometime in the past") and its dual $\mathrm{G}^{-} \varphi:=\neg \mathrm{F}^{-} \neg \varphi$ ("always in the past"). The notion of sentence is defined as for HyperCTL*. The semantics of the modalities $\mathrm{X}^{-}, \mathrm{U}^{-}$, and $\exists^{G} x$ is as follows.

$$
\begin{aligned}
& \Pi, y, i \models_{K} \mathrm{X}^{-} \varphi \Leftrightarrow i>0 \text { and } \Pi, y, i-1 \models_{K} \varphi \\
& \Pi, y, i \models_{K} \varphi_{1} \mathrm{U}^{-} \varphi_{2} \Leftrightarrow \text { for some } j \leq i: \Pi, y, j \models_{K} \varphi_{2} \text { and } \\
& \Pi, y, k \models_{K} \varphi_{1} \text { for all } j<k \leq i \\
& \Pi, y, i \models_{K} \exists^{G} x . \varphi \quad \Leftrightarrow \text { for some initial path } \pi \text { of } K, \Pi[x \leftarrow \pi], x, i \models \varphi
\end{aligned}
$$

Thus, general hyper quantification range over all the initial paths (not only the ones which visit the current node). The satisfaction relation $K=\varphi$ is defined as for HyperCTL*. Note that while the one-variable fragment of HyperCTL* corresponds to standard $\mathrm{CTL}^{*}$, the $\exists^{G}$-free one-variable fragment of HyperCTL $\mathrm{C}_{l p}^{*}$ corresponds to the well-known equi-expressive linear past memoryful extension $\mathrm{CTL}_{l p}^{*}$ of $\mathrm{CTL}^{*}$ [15]. The model-checking problem for HyperCTL ${ }_{l p}^{*}$ is checking given a finite Kripke structure $K$ and a HyperCTL ${ }_{l p}^{*}$ sentence $\varphi$, whether $K \models \varphi$. It is plain to see that HyperCTL ${ }_{l p}^{*}$ is bisimulation invariant and, in particular, $K \models \varphi$ iff $\operatorname{Unw}(K) \models \varphi$.

We consider now two relevant examples from the literature which demonstrate the expressive power of HyperCTL $\mathrm{L}_{l p}^{*}$. Both examples rely on the ability to express observational equivalence in the logic. We fix an observation map Obs. For an agent $a \in$ Agts and two paths variables $x$ and $y$ in VAR, define $\psi(a, x, y):=\mathrm{G}^{-}\left(\bigwedge_{p \in O b s(a)} p[x] \leftrightarrow p[y]\right)$

The first example shows that the logic can express distributed knowledge, a notion extensively investigated in [11]. It is crucial for information-flow security requirements as it allows to reason about adversaries who can communicate to share their knowledge: a group of agents $A \subseteq$ Agts has distributed knowledge of $\varphi$, which we will denote by $\mathrm{D}_{A} \varphi$, if the combined knowledge of the members of $A$ implies $\varphi$. It is well known that the modality $\mathrm{D}_{A}$ cannot be expressed by means of modalities $\mathrm{K}_{a}[11]$. Also, since HyperCTL* cannot express the modality $\mathrm{K}_{a}$ (see Section 3.2) and $\mathrm{K}_{a}$ is $\mathrm{D}_{\{a\}}$, it cannot express either $\mathrm{D}_{A}$. However, $\mathrm{D}_{A}$ is expressible in HyperCTL $L_{l p}^{*}$. Given a HyperCTL $\mathrm{CL}_{l p}^{*}$ formula $\varphi$, we have: $\mathrm{D}_{A} \varphi \equiv \forall^{G} y .\left[\left(\bigwedge_{a \in A} \psi(a, x, y)\right) \rightarrow \varphi\right]$. Observe that both distinctive features of HyperCTL ${ }_{l p}^{*}$ are used here: the linear past modalities to capture observational equivalence, and the general hyper quantifier to range over all the initial paths.

The second example, inspired by [1], is an opacity requirement that we conjecture can be expressed neither in HyperCTL* nor in $\mathrm{KCTL}^{*}$. Assume that agent $a$ can observe the low-security (Boolean) variables $p$ (i.e., $p \in \operatorname{Obs}(a)$ ), but not the high-security variables $q$ (i.e., $q \notin O b s(a)$ ). Consider the case of a secret 
represented by the value true of a high variable $q_{s}$. Then, the requirement $\forall x . \mathrm{G}\left(q_{s} \rightarrow \forall^{G} y . \psi(a, x, y)\right)$ says that whenever $q_{s}$ holds at some node in the computation tree, all the nodes at the same level have the same valuations of low variables. Hence, the observer $a$ cannot infer that the secret has been revealed. Here again, both the linear past and the general hyper quantifier are required.

\section{Expressiveness issues}

In this section, we establish that HyperCTL* and $\mathrm{KCTL}^{*}$ are expressively incomparable, and HyperCTL $L_{l p}^{*}$ is more expressive than both HyperCTL* and $\mathrm{KCTL}^{*}$.

Let $\mathcal{L}$ be a logic interpreted over Kripke structures, $\mathcal{L}^{\prime}$ be a logic interpreted over extended Kripke structures, and $C$ be a class of Kripke structures. For a sentence $\varphi$ of $\mathcal{L}$, a sentence $\varphi^{\prime}$ of $\mathcal{L}^{\prime}$, and an observation map Obs, $\varphi$ and $\varphi^{\prime}$ are equivalent w.r.t. $C$ and $O b s$, written $\varphi \equiv_{C, O b s} \varphi^{\prime}$ if for all Kripke structures $K \in C, K=\varphi$ iff $(K, O b s) \models \varphi^{\prime} . \mathcal{L}^{\prime}$ is at least as expressive as $\mathcal{L}$ w.r.t. $C$, written $\mathcal{L} \leq_{C} \mathcal{L}^{\prime}$, if for every sentence $\varphi$ of $\mathcal{L}$, there is an observation map Obs and a sentence $\varphi^{\prime}$ of $\mathcal{L}^{\prime}$ such that $\varphi \equiv_{C, O b s} \varphi^{\prime}$. Conversely, $\mathcal{L}$ is at least as expressive as $\mathcal{L}^{\prime}$ w.r.t. the class $C$, written $\mathcal{L}^{\prime} \leq_{C} \mathcal{L}$, if for every sentence $\varphi^{\prime}$ of $\mathcal{L}^{\prime}$ and for every observation map Obs, there is a sentence $\varphi$ of $\mathcal{L}$ such that $\varphi \equiv_{C, O b s} \varphi^{\prime}$. Note the obvious asymmetry in the above two definitions due to the fact that for evaluating a sentence in $\mathcal{L}^{\prime}$, we need to fix an observation map. If $\mathcal{L} \underline{\Sigma}_{C} \mathcal{L}^{\prime}$ and $\mathcal{L}^{\prime} \underline{Z}_{C} \mathcal{L}$, then $\mathcal{L}$ and $\mathcal{L}^{\prime}$ are expressively incomparable w.r.t. $C$. We denote by fin the class of finite Kripke structures.

\subsection{HyperCTL* is not subsumed by $\mathrm{KCTL}^{*}$}

In this section, we show that HyperCTL* and its fragment HyperLTL are not subsumed by KCTL* even if we restrict ourselves to finite Kripke structures.

\section{Theorem 1. HyperLTL $\not_{f i n} K C T L^{*}$.}

The main intuition for Theorem 1 is that unlike HyperLTL, KCTL* does not allow to relate two initial paths at an unbounded number of positions. Thus, for example, there is no mechanism in $\mathrm{KCTL}^{*}$ to select two distinct paths $\pi$ and $\pi^{\prime}$ such that the evaluations of a given LTL formula along $\pi$ and $\pi^{\prime}$ coincide at every position. Formally, in order to prove Theorem 1, we use the HyperLTL sentence of Example 2 given by $\varphi_{p}:=\exists x . \exists y . p[x] \mathrm{U}((p[x] \wedge \neg p[y]) \wedge \mathrm{XG}(p[x] \leftrightarrow p[y]))$.

We exhibit two families of regular tree structures $\left(K_{n}\right)_{n>1}$ and $\left(M_{n}\right)_{n>1}$ over $2^{\{p\}}$ such that: (i) for all $n>1, \varphi_{p}$ distinguishes between $K_{n}$ and $M_{n},{ }^{4}$ and (ii) for every $\mathrm{KCTL}^{*}$ sentence $\psi$, there is $n>1$ s.t. $\psi$ does not distinguish between $\left(K_{n}, O b s\right)$ and $\left(M_{n}, O b s\right)$ for all observation maps Obs. Hence, Theorem 1 follows.

In the following, we fix $n>1$. The regular tree structure $K_{n}$ is illustrated in Fig. 1, where $\ell_{n}>1$. Note that the root has label $\{p\}$ and $2 n+1$ successors

\footnotetext{
${ }^{4}$ i.e., $\varphi_{p}$ evaluates to true on one strucutre and to false on the other one
} 


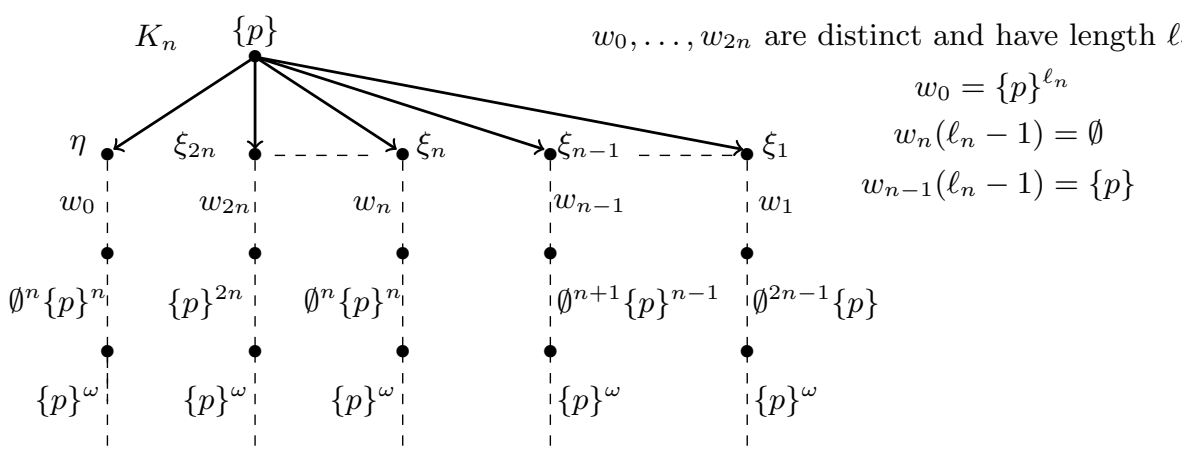

Fig. 1. The regular tree structure $K_{n}$ for the witness HyperLTL formula $\varphi_{p}$

$\eta, \xi_{1}, \ldots, \xi_{2 n}$, and there is a unique initial path visiting $\eta$ (resp., $\xi_{k}$ with $k \in$ $[1,2 n])$. We denote this path by $\pi(\eta)$ (resp., $\left.\pi\left(\xi_{k}\right)\right)$. The tree structure $M_{n}$ is obtained from $K_{n}$ by replacing the label $\{p\}$ of node $\pi\left(\xi_{n}\right)\left(\ell_{n}+1+n\right)$ with $\emptyset$. Note that in $M_{n}$, the traces of $\pi\left(\xi_{n}\right)\left[\ell_{n}+1, \infty\right]$ and $\pi\left(\xi_{n-1}\right)\left[\ell_{n}+1, \infty\right]$ coincide.

Proposition 1. $K_{n} \models \varphi_{p}$ and $M_{n} \not \models \varphi_{p}$.

Proof. In the structure $K_{n}$, the trace of the finite path $\pi(\eta)\left[0, \ell_{n}\right]$ is $\{p\}^{\ell_{n}+1}$, the label of $\pi\left(\xi_{n}\right)$ at position $\ell_{n}$ is $\emptyset$, and the traces of $\pi(\eta)\left[\ell_{n}+1, \infty\right]$ and $\pi\left(\xi_{n}\right)\left[\ell_{n}+1, \infty\right]$ coincide, which make $\pi(\eta)$ and $\pi\left(\xi_{n}\right)$ good candidates to fulfill $\varphi_{p}$. Hence, $K_{n}=\varphi_{p}$. It remains to show that $M_{n} \not \models \varphi_{p}$.

By construction, for all distinct initial paths $\pi$ and $\pi^{\prime}$ and $\ell \in\left[0, \ell_{n}\right]$, the traces of $\pi[\ell, \infty]$ and $\pi^{\prime}[\ell, \infty]$ in $M_{n}$ are distinct (recall that $\pi\left(\xi_{n}\right)\left(\ell_{n}\right)$ and $\pi\left(\xi_{n-1}\right)\left(\ell_{n}\right)$ have distinct labels). Moreover, $\pi(\eta)$ is the unique initial path of $M_{n}$ where $p$ holds at every position in $\left[0, \ell_{n}\right]$. Thus, since $\pi(\eta)\left(\ell_{n}+1\right)$ has label $\emptyset$ and there is no distinct initial path $\pi^{\prime \prime}$ of $M_{n}$ such that the traces of $\pi(\eta)\left[\ell_{n}+1, \infty\right]$ and $\pi^{\prime \prime}\left[\ell_{n}+1, \infty\right]$ coincide, by construction of $\varphi_{p}, M_{n} \not \vDash \varphi_{p}$.

A KCTL* formula $\psi$ is balanced if for every until subformula $\psi_{1} \mathrm{U} \psi_{2}$ of $\psi$, it holds that $\left|\psi_{1}\right|=\left|\psi_{2}\right|$. By using the atomic formula $T$, it is trivial to convert a $\mathrm{KCTL}^{*}$ sentence $\psi$ into an equivalent balanced $\mathrm{KCTL}^{*}$ sentence of size at most $|\psi|^{2}$. This observation together with Proposition 1, and the following non-trivial result provide a proof of Theorem 1.

Theorem 2. Let $\psi$ be a balanced $K C T L^{*}$ sentence such that $|\psi|<n$. Then, for all observation maps Obs, $\left(K_{n}, \mathrm{Obs}\right) \models \psi \Leftrightarrow\left(M_{n}, \mathrm{Obs}\right) \models \psi$.

Proof. Given an observation map Obs, it suffices to show that for all initial paths $\pi$ and positions $i \in\left[0, \ell_{n}\right], \pi, i \models_{K_{n}, O b s} \psi$ iff $\pi, i \models_{M_{n}, O b s} \psi$. The key for obtaining this result is that since $|\psi|<n, \psi$ cannot distinguish the nodes $\pi\left(\xi_{n}\right)\left(\ell_{n}+1\right)$ and $\pi\left(\xi_{n-1}\right)\left(\ell_{n}+1\right)$ both in $\left(K_{n}, O b s\right)$ and in $\left(M_{n}, O b s\right)$. For $M_{n}$, this indistinguishability easily follows from the construction and is independent 
of the size of $\psi$. For $K_{n}$, the indistinguishability is non-trivial and is formally proved by defining equivalence relations on the set of nodes at distance $d \in\left[\ell_{n}+\right.$ $\left.1, \ell_{n}+2 n\right]$ from the root, which are parameterized by a natural number $h \in[1, n]$, where $h$ intuitively represents the size of the current balanced subformula of $\psi$ in the recursive evaluation of $\psi$ on $K_{n}$.

\subsection{KCTL* is not subsumed by HyperCTL*}

In this section, we show that $\mathrm{KCTL}^{*}$ and its fragment $\mathrm{KLTL}$ are not subsumed by HyperCTL* even with respect to finite Kripke structures. The intuitive insight is that unlike KLTL, HyperCTL* cannot express requirements which relate at some position an unbounded number of paths.

For $p \in \mathrm{AP}$, an observation map Obs is $p$-blind if for all agents $a, p \notin O b s(a)$.

Theorem 3. KLTL $\not_{f i n} H y p e r C T L^{*}$.

As witness KLTL sentence for Theorem 3, we use the KLTL sentence of Example 1 given by $\varphi_{p}:=\forall \mathrm{XFK}_{a} \neg p$. We exhibit two families of regular tree structures $\left(K_{n}\right)_{n>1}$ and $\left(M_{n}\right)_{n>1}$ over $2^{\{p\}}$ such that the following holds for all $n>1$ : (i) for each $p$-blind observation map Obs, $\varphi_{p}$ distinguishes between $\left(K_{n}, O b s\right)$ and $\left(M_{n}, O b s\right)$, and (ii) no HyperCTL* formula $\psi$ of size less than $n$ distinguishes between $K_{n}$ and $M_{n}$. Hence, Theorem 3 follows.

Fix $n>1$. In order to define $K_{n}$ and $M_{n}$, we need additional definitions.

An $n$-block is a word in $\{p\} \emptyset^{*}$ of length at least $n+2$. Given finite words $w_{1}, \ldots, w_{k}$ over $2^{\{p\}}$ having the same length $\ell$, the join join $\left(w_{1}, \ldots, w_{k}\right)$ of $w_{1}, \ldots, w_{k}$ is the word of length $\ell$ such that $j o i n\left(w_{1}, \ldots, w_{k}\right)(i)=w_{1}(i) \cup \ldots \cup$ $w_{k}(i)$ for all $i \in[0, \ell-1]$. For a finite word $w$ over $2^{\{p\}}$, the dual $\widetilde{w}$ of $w$ is the word over $2^{\{p\}}$ of length $|w|$ such that for all $i \in[0,|w|-1], p \in \widetilde{w}(i)$ iff $p \notin w(i)$.

Given $n$ finite words $w_{1}, \ldots, w_{n}$ over $2^{\{p\}}$ of the same length, the tuple $\left\langle w_{1}, \ldots, w_{n}\right\rangle$ satisfies the $n$-fractal requirement if for all $k \in[1, n]$,

$$
j o i n\left(w_{1}, \ldots, w_{k}\right) \text { is of the form } b l_{1}^{k} \ldots b l_{m_{k}}^{k} \cdot\{p\}
$$

where $b l_{1}^{k} \ldots b l_{m_{k}}^{k}$ are $n$-blocks. Moreover, $m_{1}=n+4$, and the following holds: if $k<n$, then $w_{k+1}$ is obtained from join $\left(w_{1}, \ldots, w_{k}\right)$ by replacing the last symbol with $\emptyset$, and by replacing each $n$-block $b l_{i}^{k}$ of $j o i n\left(w_{1}, \ldots, w_{k}\right)$ by a sequence of $n+4 n$-blocks preceded by a non-empty word in $\emptyset^{*}$ of length at least $n+2$.

Remark 1. Assume that $\left\langle w_{1}, \ldots, w_{n}\right\rangle$ satisfies the $n$-fractal requirement and let $\ell$ be the common length of $w_{1}, \ldots, w_{n}$. Then, for all $i \in[0, \ell-1]$, there is at most one $k \in[1, n]$ such that $p \in w_{k}(i)$. Moreover, $p \in w_{1}(0)$ and $p \in w_{1}(\ell-1)$.

Definition 1 (The tree structures $K_{n}$ and $M_{n}$ ). $K_{n}$ is illustrated in Fig. 2 where $\ell_{n}>1$. The unique initial path visiting node $\eta$ (resp., $\xi_{k}$ with $k \in[1, n]$ ) is denoted by $\pi(\eta)$ (resp., $\pi\left(\xi_{k}\right)$ ).

$A$ main position is a position in $\left[1, \ell_{n}\right]$. Let $i_{\text {alert }}$ be the third (in increasing order) main position $i$ along $\pi\left(\xi_{1}\right)$ such that the label of $\pi\left(\xi_{1}\right)(i)$ in $K_{n}$ is $\{p\}$ (note that $i_{\text {alert }}$ exists). Then, the regular tree structure $M_{n}$ is obtained from $K_{n}$ by replacing the label $\{p\}$ of $\pi\left(\xi_{1}\right)$ at position $i_{\text {alert }}$ with $\emptyset$. 


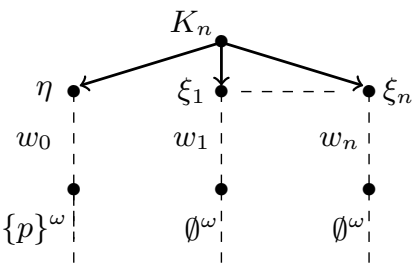

Fig. 2. The regular tree structure $K_{n}$ for the witness $\mathrm{KLTL}$ formula $\varphi_{p}:=\forall \mathrm{XFK}_{a} \neg p$

By construction, in the tree structure $K_{n}$, for each non-root level, there is a node where $p$ holds and a node where $p$ does not hold. Hence, $\left(K_{n}, O b s\right) \not \models \varphi_{p}$. By Remark 1, for each main position $i$, there is at most one $k \in[1, n]$ such that the label of $\pi\left(\xi_{k}\right)(i)$ in $K_{n}$ is $\{p\}$. If such a $k$ exists, we say that $i$ is a main $p$-position and $\xi_{k}$ is the type of $i$. Now, for the level of $M_{n}$ at distance $i_{\text {alert }}$ from the root, $p$ uniformly does not hold (i.e., there is no node of $M_{n}$ at distance $i_{\text {alert }}$ from the root where $p$ holds). Thus, we obtain the following result.

Proposition 2. For each p-blind observation map Obs, $\left(K_{n}, O b s\right) \not=\varphi_{p}$ and $\left(M_{n}, O b s\right) \models \varphi_{p}$.

Theorem 3 directly follows from Proposition 2 and the following result.

Theorem 4. For all HyperCTL* sentences $\psi$ with $|\psi|<n, K_{n} \models \psi \Leftrightarrow M_{n} \models \psi$.

Proof. The main idea is that for a HyperCTL* sentence $\psi$ of size less than $n$, in the recursive evaluation of $\psi$ on the tree structure $M_{n}$, there will be $h_{*} \in[2, n]$ such that the initial path $\pi\left(\xi_{h_{*}}\right)$ is not bound by the current path assignment. Then, the $n$-fractal requirement ensures that in $M_{n}$, the main $p$-position $i_{\text {alert }}$ (which in $M_{n}$ has label $\emptyset$ along $\left.\pi\left(\xi_{1}\right)\right)$ is indistinguishable from the main $p$-positions $j$ of type $\xi_{h_{*}}$ which are sufficiently 'near' to $i_{\text {alert }}$ (such positions $j$ have label $\emptyset$ along the initial paths $\pi\left(\xi_{k}\right)$ with $\left.k \neq h *\right)$. We formalize this intuition by defining equivalence relations on the set of main positions which are parameterized by $h_{*}$ and a natural number $\mathfrak{m} \in[0, n]$ and reflect the fractal structure of the main $p$-position displacement. Since the number of main $p$-positions of type $\xi_{1}$ following $i_{\text {alert }}$ is at least $n$, we then deduce that in all the positions $i$ such that $i \leq i_{F}$, where $i_{F}$ is the main $p$-position of type $\xi_{1}$ preceding $i_{\text {alert }}$, no HyperCTL* formula $\psi$ can distinguish $M_{n}$ and $K_{n}$ with respect to path assignments such that $|\Pi|+|\psi|<n$, where $|\Pi|$ is the number of initial paths bound by $\Pi$. Hence, the result follows.

\subsection{HyperCTL $L_{l p}^{*}$ unifies $\mathrm{KCTL}^{*}$ and HyperCTL*}

We show that $\mathrm{KCTL}^{*}$ can be easily translated in linear time into the two-variable fragment of HyperCTL $L_{l p}^{*}$. Intuitively, the knowledge modalities can be simulated by the general hyper path quantifiers combined with the temporal past modalities. Hence, we obtain the following result. 
Theorem 5. Given a $K C T L^{*}$ sentence $\psi$ and an observation map Obs, one can construct in linear time a HyperCTL $L_{l p}^{*}$ sentence $\varphi$ with just two path variables such that for each Kripke structure $K, K \models \varphi \Leftrightarrow(K, O b s) \models \psi$.

Note that the $\mathrm{KCTL}^{*}$ sentence $\forall \mathrm{XFK}_{a} \neg p$ used to prove Theorem 3 is equivalent w.r.t. $p$-blind observation maps to the HyperCTL $L_{l p}^{*}$ sentence $\forall x . \operatorname{XF}\left(\forall^{G} y . \neg p[y]\right)$ which does not use past modalities. Thus, by Theorems 1, 3, and 5, we obtain:

Corollary 1. HyperCTL $L_{l p}^{*}$ is more expressive than both HyperCTL* and $K C T L^{*}$. Moreover, the future fragment of HyperCTL $L_{l p}^{*}$ (where past-time modalities are disallowed) is already more expressive than HyperCTL*.

\section{Model-checking against HyperCTL ${ }_{l p}^{*}$}

In this section, we address the model-checking problem for HyperCTL ${ }_{l p}^{*}$. Similarly to the proof given in [6] for the less expressive logic HyperCTL*, we show that the above problem is non-elementarily decidable by linear-time reductions from/to satisfiability of full Quantified Propositional Temporal Logic (QPTL, for short) [23], which extends LTL with past (PLTL) by quantification over propositions. As main contribution of this section, we address complexity issues for the considered problem by providing optimal complexity bounds in terms of a parameter of the given HyperCTL $L_{l p}^{*}$ formula, we call strong alternation depth. For this, we first provide similar optimal complexity bounds for satisfiability of QPTL. As a corollary of our results, we also obtain that for a relevant fragment of HyperCTL ${ }_{l p}^{*}$, model-checking is EXPSPACE-complete. With regard to QPTL, well-known optimal complexity bounds, in terms of the alternation depth of existential and universal quantifiers, concern the fragment of QPTL in prenex form (quantifiers cannot occur in the scope of temporal modalities) [23]. QPTL formulas can be translated in polynomial time into equisatisfiable QPTL formulas in prenex form, but in this conversion, the nesting depth of temporal modalities in the original formula (in particular, the alternation depth between always and eventually modalities and the nesting depth of until modalities) lead to an equal increasing in the quantifier alternation depth of the resulting formula. We show that this can be avoided by directly applying a non-trivial automatic theoretic approach to unrestricted QPTL formulas. Our results also improve in a meaningful way the upper bounds provided in [6] for model-checking of HyperCTL*; indeed, in [6], differently from our approach, occurrences of temporal modalities count as additional alternations.

The logic QPTL [23]. QPTL formulas $\varphi$ over AP are defined as follows:

$$
\varphi::=\top|p| \neg \varphi|\varphi \wedge \varphi| \mathrm{X} \varphi\left|\mathrm{X}^{-} \varphi\right| \varphi \mathrm{U} \varphi\left|\varphi \mathrm{U}^{-} \varphi\right| \exists p . \varphi
$$

where $p \in \mathrm{AP}$. The positive normal form of a QPTL formula $\varphi$ is obtained by pushing inward negations to propositional literals using De Morgan's laws and the duals $\mathrm{R}$ (release), $\mathrm{R}^{-}$(past release), and $\forall p$ (propositional universal quantifier) of $\mathrm{U}^{-} \mathrm{U}^{-}$, and $\exists p$, respectively. A formula is (pure) existential if its positive normal has no universal quantifier. Analogous notions apply to HyperCTL ${ }_{l p}^{*}$. 
QPTL formulas are interpreted over (infinite) pointed words $(w, i)$ over $2^{\mathrm{AP}}$ consisting of an infinite word $w$ over $2^{\mathrm{AP}}$ and a position $i \geq 0$. The semantics of propositional quantification is as follows.

$(w, i) \models \exists p . \varphi \Leftrightarrow$ there is $w^{\prime} \in\left(2^{\mathrm{AP}}\right)^{\omega}$ such that $w=\mathrm{AP} \backslash\{p\} w^{\prime}$ and $\left(w^{\prime}, i\right) \models \varphi$ where $w={ }_{\mathrm{AP} \backslash\{p\}} w^{\prime}$ means that the projections of $w$ and $w^{\prime}$ over $\mathrm{AP} \backslash\{p\}$ coincide. For a QPTL formula $\varphi$, let $\mathcal{L}_{\wp}(\varphi)$ be the set of pointed words satisfying $\varphi$, and $\mathcal{L}(\varphi)$ be the set $\left\{w \mid(w, 0) \in \mathcal{L}_{\wp}(\varphi)\right\} ; \varphi$ is satisfiable if $\mathcal{L}(\varphi) \neq \emptyset$.

Optimal bounds for QPTL satisfiability. First, we give a generalization of the standard notion of alternation depth between existential and universal quantifiers, we call strong alternation depth. This notion takes into account also the occurrence of temporal modalities between quantifier occurrences, but the nesting depth of temporal modalities is not considered (it is collapsed to one).

Definition 2. Let $\mathcal{O}=\left\{\exists, \forall, U, U^{-}, R, R^{-}, G, G^{-}, F, F^{-}\right\}$. First, we define the strong alternation length $\ell(\chi)$ of finite sequences $\chi \in \mathcal{O}^{*}: \ell(\varepsilon)=0, \ell(O)=1$ for all $O \in \mathcal{O}$, and

$$
\ell\left(O O^{\prime} \chi\right)= \begin{cases}\ell\left(O^{\prime} \chi\right) & \text { if } O^{\prime} \in \mathcal{O} \backslash\{\exists, \forall\} \\ \ell\left(O^{\prime} \chi\right) & \text { if either } O, O^{\prime} \in\{\exists, F, F\} \text { or } O, O^{\prime} \in\left\{\forall, G, G^{-}\right\} \\ 1+\ell\left(O^{\prime} \chi\right) & \text { otherwise }\end{cases}
$$

${ }^{5}$ Then, the strong alternation depth $\operatorname{sad}(\varphi)$ of a QPTL formula $\varphi$ is the maximum over the strong alternation lengths $\ell(\chi)$, where $\chi$ is the sequence of modalities in $\mathcal{O}$ along a path in the tree encoding of the positive normal form of $\varphi$. The strong alternation depth $\operatorname{sad}(\varphi)$ of a HyperCTL $L_{l p}^{*}$ formula $\varphi$ is defined similarly but we replace quantification over propositions with quantification over path variables. For a QPTL (resp., HyperCTL $L_{l p}^{*}$ ) formula $\varphi$, if there is a subformula $\psi$ of the positive normal form of $\varphi$ whose root operator is a universal quantifier and such that $\operatorname{sad}(\psi)=\operatorname{sad}(\varphi)$, then we say that $\varphi$ is a first-level universal formula; otherwise, we say that $\varphi$ is a first-level existential formula.

Note that for a QPTL formula $\varphi$ in prenex form, the strong alternation depth corresponds to the alternation depth of existential and universal quantifiers plus one. For all $n, h \in \mathbb{N}$, Tower $(h, n)$ denotes a tower of exponentials of height $h$ and argument $n$ : $\operatorname{Tower}(0, n)=n$ and $\operatorname{Tower}(h+1, n)=2^{\operatorname{Tower}(h, n)}$. We establish the following result, where $h$-EXPSPACE is the class of languages decided by deterministic Turing machines bounded in space by functions of $n$ in $O\left(\operatorname{Tower}\left(h, n^{c}\right)\right)$ for some constant $c \geq 1$.

Theorem 6. For all $h \geq 1$, satisfiability of QPTL formulas $\varphi$ with strong alternation depth at most $h$ is $h$-EXPSPACE-complete, and $(h-1)$-EXPSPACEcomplete in case $\varphi$ is first-level existential or pure existential (even if we only allow temporal modalities in $\left\{X, X^{-}, F, F, G, G^{-}\right\}$).

\footnotetext{
$\overline{{ }^{5} \text { For example }}, \ell(\exists \mathrm{GU} \exists \mathrm{U})=\ell(\mathrm{U} \exists \mathrm{U})=2$.
} 
Here, we illustrate the upper bounds of Theorem 6. In the automata-theoretic approach for QPTL formulas $\varphi$ in prenex form, first, one converts the quantifierfree part $\psi$ of $\varphi$ into an equivalent Büchi nondeterministic automaton (Büchi NWA) accepting $\mathcal{L}(\psi)$. Then, by using the closure of Büchi NWA definable languages under projection and complementation, one obtains a Büchi NWA accepting $\mathcal{L}(\varphi)$. This approach would not work for arbitrary QPTL formulas $\varphi$, where quantifiers can occur in the scope of temporal modalities. In this case, for a subformula $\varphi^{\prime}$ of $\varphi$, we need to keep track of the full set $\mathcal{L}_{\wp}\left(\varphi^{\prime}\right)$ of pointed words satisfying $\varphi$, and not simply $\mathcal{L}\left(\varphi^{\prime}\right)$. Thus, we resort to two-way automata $\mathcal{A}$ accepting languages $\mathcal{L}_{\wp}(\mathcal{A})$ of pointed words. In particular, the proposed approach is based on a compositional translation of QPTL formulas into a simple two-way extension of Büchi NWA, which we call Büchi SNWA. Essentially, given an input pointed word $(w, i)$, a Büchi SNWA splits in two copies: the first one moves forward along the suffix $w[i, \infty]$ and the second one moves backward along the prefix $w[0, i]$.

Moreover, at each step of the translation into Büchi SNWA, we use as an intermediate formalism a two-way extension of the class of (one-way) hesitant alternating automata (HAA, for short) over infinite words introduced in [17]. Like one-way HAA, the set of states $Q$ of a two-way HAA is partitioned into a set of components $Q_{1}, \ldots, Q_{n}$ such that moves from states in $Q_{i}$ lead to states in components $Q_{j}$ so that $j \leq i$. Moreover, each component is classified as either past, or Büchi, or coBüchi: in a past (resp., Büchi/coBüchi) component $Q_{i}$, the unique allowed moves from $Q_{i}$ to $Q_{i}$ itself are backward (resp., forward). These syntactical requirements ensure that in a run over a pointed word, every infinite path $\pi$ of the run gets trapped in some Büchi or coBüchi component, and the path $\pi$ eventually use only forward moves. Moreover, the acceptance condition of a two-way HAA encodes a particular kind of parity condition of index 2: a Büchi/coBüchi component $Q_{i}$ has an associated subset $F_{i} \subseteq Q_{i}$ of accepting states. Then, a run is accepting if for every infinite path $\pi$, denoting with $Q_{i}$ the Büchi/coBüchi component in which $\pi$ gets trapped, $\pi$ satisfies the Büchi/coBüchi acceptance condition associated with $Q_{i}$. For two-way HAA $\mathcal{A}$, we establish two crucial results. First, the dual automaton $\widetilde{\mathcal{A}}$ obtained from $\mathcal{A}$ by dualizing the transition function, and by converting a Büchi (resp., coBüchi) component into a coBüchi (resp., Büchi) component is still a two-way HAA. Thus, by standard arguments (see e.g. [25]), automaton $\widetilde{\mathcal{A}}$ accepts the complement of $\mathcal{L}_{\wp}(\mathcal{A})$. Second, by using the notion of odd ranking function for standard coBüchi alternating automata [16] (which allows to convert a coBüchi acceptance condition into a Büchi-like acceptance condition) and a non-trivial generalization of the Miyano-Hayashi construction [20], we show that two-way HAA can be converted in singly exponential time into equivalent Büchi SNWA.

Theorem 7. Given a two-way $H A A \mathcal{A}$ with $n$ states, the following holds:

1. the dual automaton $\widetilde{\mathcal{A}}$ of $\mathcal{A}$ is a two-way $H A A$ accepting the complement of $\mathcal{L}_{\wp}(\mathcal{A})$;

2. one can build "on the fly" and in singly exponential time a Büchi SNWA accepting $\mathcal{L}_{\wp}(\mathcal{A})$ with $2^{O(n \cdot \log (n))}$ states. 
Finally, by using Theorem 7, we establish the following result from which the upper bounds of Theorem 6 directly follow (note that Büchi SNWA $\mathcal{A}$ can be trivially converted into Büchi NWA accepting the set of infinite words $w$ such that $(w, 0) \in \mathcal{L}_{\wp}(\mathcal{A})$, and checking non-emptiness for Büchi NWA is in NLOGSPACE).

Theorem 8. Let $\varphi$ be a first-level existential (resp., first-level universal) QPTL formula and $h=\operatorname{sad}(\varphi)$. Then, one can construct "on the fly" a Büchi SNWA $\mathcal{A}_{\varphi}$ accepting $\mathcal{L}_{\wp}(\varphi)$ in time $\operatorname{Tower}(h, O(|\varphi|))$ (resp., Tower $\left.(h+1, O(|\varphi|))\right)$.

Proof. By structural induction on the positive normal form $\varphi_{+}$of $\varphi$. The relevant case is when the outermost operator of $\varphi_{+}$is a temporal modality (the other cases easily follow from Theorem 7 and the closure of Büchi SNWA definable pointed languages under union, intersection, and projection). This case is handled by first building a two-way HAA $\mathcal{A}$ accepting $\mathcal{L}_{\wp}(\varphi)$ and then by applying Theorem $7(2)$. The construction of $\mathcal{A}$ is obtained by a generalization of the standard linear-time translation of LTL formulas into Büchi alternating automata which exploits the (inductively built) Büchi SNWA associated with the maximal quantified subformulas of $\varphi_{+}$.

Optimal bounds for model-checking of HyperCTL ${ }_{l p}^{*}$. By establishing lineartime reductions from/to satisfiability of QPTL and by exploiting Theorem 6 , we provide optimal bounds on the complexity of model-checking for $\mathrm{HyperCTL}_{l p}^{*}$ in terms of the strong alternation depth of the formula. In particular, the lineartime reduction to satisfiability of QPTL generalizes the one given in [6] for the model checking of HyperCTL*.

Theorem 9. For all $h \geq 1$ and HyperCTL $L_{l p}^{*}$ sentences $\varphi$ with strong alternation depth at most $h$, model-checking against $\varphi$ is $h$-EXPSPACE-complete, and $(h-$ 1)-EXPSPACE-complete in case $\varphi$ is first-level existential or pure existential (even if we allow only temporal modalities in $\left\{X, X^{-}, F, F, G, G^{-}\right\}$).

By Theorem 9, for the first-level existential fragment $\mathcal{F}$ of HyperCTL ${ }_{l p}^{*}$ where the strong alternation depth is at most 2, model-checking is EXPSPACEcomplete. Notice that the HyperCTL* fragment $\mathcal{F}^{\prime}$ of $\mathcal{F}$ can express important classes of information-flow requirements as illustrated in [6], and that the modelchecking algorithm in [6] applied to $\mathcal{F}^{\prime}$ leads to a non-elementary upper bound.

\section{Discussion}

We plan to extend this work in many directions. First, we intend to identify tractable fragments of HyperCTL ${ }_{l p}^{*}$ and to investigate their synthesis problem; note that satisfiability of HyperCTL* is already undecidable [6]. Second, we should extend the framework to deal with asynchronicity, as information flows are relevant for security in many asynchronous frameworks, such as distributed systems or cryptographic protocols. In the same line, we would like to investigate the possibility of extending the verification of information-flow requirements to relevant classes of infinite-state systems such as the class of pushdown systems, a model extensively investigated in software verification. 


\section{References}

1. R. Alur, P. Černỳ, and S. Chaudhuri. Model checking on trees with path equivalences. In Proc. 13th TACAS, LNCS 4424, pages 664-678. Springer, 2007.

2. R. Alur, P. Cerný, and S. Zdancewic. Preserving secrecy under refinement. In Proc. 33rd ICALP, LNCS 4052, pages 107-118. Springer, 2006.

3. M. Balliu, M. Dam, and G. L. Guernic. Epistemic temporal logic for information flow security. In Proc. PLAS, page 6. ACM, 2011.

4. L. Bozzelli, B. Maubert, and S. Pinchinat. Unifying hyper and epistemic temporal logic. CoRR, abs/1409.2711, 2014.

5. J. Bryans, M. Koutny, L. Mazaré, and P. Ryan. Opacity generalised to transition systems. Int. J. Inf. Sec., 7(6):421-435, 2008.

6. M. Clarkson, B. Finkbeiner, M. Koleini, K. Micinski, M. Rabe, and C. Sánchez. Temporal logics for hyperproperties. In Proc. 3rd POST, LNCS 8414, pages 265284. Springer, 2014.

7. M. Clarkson and F. Schneider. Hyperproperties. Journal of Computer Security, 18(6):1157-1210, 2010.

8. C. Dima. Revisiting satisfiability and model-checking for CTLK with synchrony and perfect recall. In Proc. 9th CLIMA, LNCS 5405, pages 117-131. Springer, 2008.

9. R. Dimitrova, B. Finkbeiner, M. Kovács, M. Rabe, and H. Seidl. Model checking information flow in reactive systems. In Proc. 13th VMCAI, LNCS 7148, pages 169-185. Springer, 2012.

10. E. Emerson and J. Halpern. "Sometimes" and "Not Never" revisited: on branching versus linear time temporal logic. J. ACM, 33(1):151-178, 1986.

11. R. Fagin, J. Halpern, and M. Vardi. Reasoning about knowledge, volume 4. MIT press Cambridge, 1995.

12. J. Goguen and J. Meseguer. Security policies and security models. In IEEE Symposium on Security and privacy, volume 12, 1982.

13. J. Halpern and K. O'Neill. Secrecy in multiagent systems. ACM Trans. Inf. Syst. Secur., 12(1), 2008.

14. J. Halpern, R. van der Meyden, and M. Vardi. Complete Axiomatizations for Reasoning about Knowledge and Time. SIAM J. Comput., 33(3):674-703, 2004.

15. O. Kupferman, A. Pnueli, and M. Vardi. Once and for all. J. Comput. Syst. Sci., 78(3):981-996, 2012.

16. O. Kupferman and M. Vardi. Weak alternating automata are not that weak. $A C M$ Transactions on Computational Logic, 2(3):408-429, 2001.

17. O. Kupferman, M. Vardi, and P. Wolper. An Automata-Theoretic Approach to Branching-Time Model Checking. J. ACM, 47(2):312-360, 2000.

18. J. McLean. Proving noninterference and functional correctness using traces. Journal of Computer Security, 1(1):37-58, 1992.

19. D. Milushev and D. Clarke. Towards incrementalization of holistic hyperproperties. In Proc. 1st POST, LNCS 7215, pages 329-348. Springer, 2012.

20. S. Miyano and T. Hayashi. Alternating finite automata on $\omega$-words. Theoretical Computer Science, 32:321-330, 1984.

21. A. Pnueli. The temporal logic of programs. In Proc. 18th FOCS, pages 46-57. IEEE Computer Society, 1977.

22. N. Shilov and N. Garanina. Model checking knowledge and fixpoints. In Proc. FICS, BRICS Notes Series, pages 25-39, 2002.

23. A. Sistla, M. Vardi, and P. Wolper. The complementation problem for Büchi automata with appplications to temporal logic. Theoretical Computer Science, 49:217-237, 1987.

24. R. van der Meyden and N. Shilov. Model checking knowledge and time in systems with perfect recall (extended abstract). In Proc. 19th FSTTCS, LNCS 1738, pages 432-445. Springer, 1999.

25. W. Zielonka. Infinite games on finitely coloured graphs with applications to automata on infinite trees. Theoretical Computer Science, 200(1-2):135-183, 1998. 